\title{
De l'épaisseur du discours : horizontalement, verticalement... et dans tous les sens
}

\author{
Jacques Bres \\ Praxiling, Montpellier III
}

\section{Introduction}

Les notes qui suivent peuvent être considérées comme tour réactif à la lecture, certainement trop rapide et superficielle, (i) des communications retenues dans l'axe Discours, pragmatique, interaction, et (ii) de Goodwin 2007. Je qualifierai le point de vue théorique dont elles procèdent comme de biais mais pas forcément biaisé: il est celui d'un pratiquant dilettante de l'analyse du discours (désormais AD), notamment «canal historique» (Rosier et Paveau 2005), comme de l'analyse conversationnelle (désormais AC) dans ses différentes formes, qui trouve encore son bonheur dans la lecture des travaux de Bakhtine, et qui s'intéresse surtout actuellement à l'actualisation de la langue en discours et au dialogisme... Lesdites notes, au-delà du questionnement, invitent au débat.

\section{Discours : dialogalité, monologalité}

Premier constat : alors que le premier terme de l'intitulé de notre axe d'étude «Discours, pragmatique, interaction » est discours, force est de constater qu'a été fortement privilégié un seul type de discours (corpus, problématiques, références bibliographiques) : le discours dialogal (alternance de locuteurs selon l'alternance des tours ; interaction in praesentia, au moins temporellement), au détriment du discours monologal (absence d'alternance de locuteurs / de tours; interaction in absentia). Comme si les recherches actuelles ${ }^{1}$ tordaient le bâton du discours et de son étude dans le sens inverse de la torsion que lui avait infligée l'AD des années 1970-1980, qui n'a guère travaillé que le discours monologal.

\section{Discours : verticalité, horizontalité}

Cette évolution dans le choix des corpus - tendanciellement du monologal au dialogal - qui s'initie au cours des années 1980-1990 et que confirme notre colloque (du moins au regard des communications retenues), s'accompagne d'un changement d'approche radical : le discours, dans l'AD, était considéré dans sa profondeur verticale inconsciente, selon laquelle le locuteur est dit plus qu'il ne dit, voire répète à son insu du déjà dit. Le discours est, dans l'approche de l'AC, traité comme surface horizontale cogérée et coproduite par les interactants : le locuteur dit avec son / ses interlocuteur(s). En simplifiant quelque peu, on peut avancer que l'AD privilégiait fortement l'interdiscursvité et négligeait l'interlocution. Dans quelle mesure les travaux actuels en $\mathrm{AC}$ en privilégiant l'interlocution, ne négligent-ils pas l'interdiscursivité?

On pourrait rendre compte de la focalisation sur la profondeur verticale pour l'AD, comme de la focalisation sur la surface horizontale pour l'AC, par les différences de corpus : le monologal, plus formel, se construirait principalement de son rapport aux autres discours; le dialogal, plus familier surtout s'il est conversationnel, se construirait principalement de son rapport aux autres locuteurs. Cette analyse, si elle contient une part de vérité, ne me parait pas fondamentalement juste; et d'autre part, elle laisse de côté la question du choix des corpus. Il y a là une question d'importance : dis-moi ce sur quoi tu travailles et avec quels outils, et je te dirai qui tu es, ou plutôt qui tu crois être, à savoir à quelle idéologie ton travail participe, le plus souvent à ton insu... 
Je ne fais que pointer ce fait, sans le développer ici. Je voudrais plutôt questionner l'AC et son approche horizontale de la production du discours à partir d'un autre paradigme théorique : celui du dialogisme, issu des travaux du cercle de Bakhtine ${ }^{2}$.

\section{Discours : dialogal et dialogique}

S'il n'est pas question de rapprocher trop facilement l'AD et les travaux de Bakhtine (cf., pour une mise en garde, Authier 1982), et encore moins d'assimiler les notions d'interdiscours et de dialogisme (cf. Bres et Rosier 2008), il me semble que les deux approches posent la question de la dimension verticale du discours, que semble oublier l'AC.

Rappelons brièvement quelques faits: Bakhtine/Voloshinov 1929 consacre un chapitre entier à l'interaction verbale et propose un ordre pour l'étude des faits linguistiques qui anticipe largement sur l'approche interactionnelle des faits linguistiques :

L'ordre méthodologique pour l'étude de la langue doit être le suivant :

1. les formes et les types d'interaction verbale en liaison avec les conditions concrètes où celleci se réalise ;

2. les formes des énonciations distinctes, des actes de parole isolés, en liaison étroite avec l'interaction dont ils constituent les éléments, c'est-à-dire les catégories d'actes de parole dans la vie et dans la création idéologique qui se prêtent à une détermination par l'interaction verbale ;

3. A partir de là, examen des formes de la langue dans leur interprétation linguistique habituelle (1929: 137)

Cependant, dans ce même ouvrage, à l'heure de passer à l'étude d'un phénomène concret, c'est le discours rapporté qui est choisi comme objet d'analyse, soit un fait discursif qui met en jeu, plutôt que la dimension dialogale horizontale, la dimension dialogique verticale. Rappelons que la distinction terminologique dialogal / dialogique, largement circulante aujourd'hui ${ }^{3}$, n'est pas de Bakhtine lui-même mais procède directement de sa problématique (Bres 2005) :

- la dimension dialogale (cf. dialogalité, supra 1.) concerne le dialogue externe, c'est-à-dire tout ce qui a trait au dialogue en tant qu'alternance des tours de deux ou plusieurs interlocuteurs, et se passe sur le fil du discours ;

- la dimension dialogique concerne le dialogue interne. Elle tient à l'orientation de tout discours vers d'autres discours et ce, triplement : (i) interdiscursivement, le discours en train de se faire ne peut pas ne pas rencontrer les autres discours qui, avant lui, se sont saisis du même objet, ni entrer en interaction avec eux; les mots sont d'autre part toujours habités des sens de ces autres discours, avec lesquels également l'interaction est incontournable ; (ii) interlocutivement, le discours en train de se faire ne peut pas ne pas anticiper sur la réception - en tant qu'énoncé-réponse - que le locuteur imagine par avance que son interlocuteur en fera ${ }^{4}$; (iii) intralocutivement: la production du discours se fait constamment en interaction avec ce que le locuteur a dit antérieurement, et avec ce qu'il envisage de dire. Cette triple orientation est à l'origine d'une triple interaction verticale, qui a pour résultat la dialogisation intérieure du discours produit, dont les manifestations sont extrêmement variées : de la citation explicite (discours direct, îlot textuel...) à des phénomènes d'échos, de résonances, d'harmoniques fort subtils. On parle de la pluralité des voix (terme métaphorique à comprendre, selon les théories, comme 'énonciateurs', ou ' points de vue') qui feuillètent tout énoncé depuis sa macrostructure (le roman, le texte, le discours, le tour de parole) jusqu'à sa microstructure : le mot.

Les travaux de Bakhtine privilégient des objets d'étude relevant du dialogique, comme p. ex. le discours rapporté, et ne travaillent pas vraiment le dialogal. Ceci pourrait expliquer la relative ignorance de la problématique dialogique par l'AC. Pourtant la dimension dialogique, notamment interdiscursive, n'intervient-elle pas sur le fil du discours? L'interaction verticale avec les discours antérieurs n'est-elle pas un paramètre de la production horizontale du discours-en-interaction avec l'interlocuteur? N'y a-t-il 
pas une pertinence du dialogique pour l'AC? C'est la cause que je voudrais brièvement plaider. Non pas théoriquement, mais pratiquement à partir de quelques occurrences de discours concrètes, prises dans les travaux conversationalistes.

\section{Oublié Bakhtine?}

Goodwin (2007) est, à ma connaissance ${ }^{5}$, un des rares travaux conversationalistes ${ }^{6}$ à dialoguer avec les recherches du cercle de Bakhtine; encore ne le fait-il qu'avec Bakhtine/Voloshinov 1929. L'auteur fait remarquer fort justement que Voloshinov tout comme Goffman (1981) développent une conception des instances de l'interlocution qui, tout occupée à définir la complexité des rôles du locuteur, en oublie de poser, parallèlement, la complexité des rôles interactifs de l'interlocuteur (hearer). Goodwin appuie sa démonstration par l'analyse de fragments d'interaction dans lesquels intervient le discours rapporté ${ }^{7}$. Je prends un exemple de ces analyses, fort pertinentes, pour pointer l'oubli qui s'y manifeste, selon moi, de la dimension dialogique verticale.

(1) Une femme raconte à un couple d'amis, en présence de son mari Don, une bourde verbale de celui-ci lors d'une interaction antérieure avec d'autres amis (Goodwin op. cit. : 4) :

13 Ann : $\quad \operatorname{Do}(\mathrm{h})^{8} \mathrm{n}$ said $(0.3)$

14

dih-did they ma:ke you take this-wa(h)llpa(h)per?

15Beth :

hh!

16Ann : $\quad=$ er(h)di-dju $\quad$ pi(h)ck i(h)t ou(h)t

Goodwin, à partir de l'étude de la vidéo, montre qu'un interlocuteur participe activement, par sa mimogestualité au récit de Ann : il s'agit très précisément de celui dont la parole est rapportée, à savoir Don, le mari de la narratrice, dont le visage et le corps accompagnent par des signaux de rire le rire de sa femme rapportant sa bourde en 14-16. D'une façon très fine, l'auteur souligne que le discours de Ann, à partir de (13) $(D o(h) n$ said $)$ permet à Don de faire une projection et de doubler par la mimique gestuelle la vocalité $\mathrm{du}$ rire de sa femme. Question naïve: la projection que fait Don se fonde-t-elle seulement sur l'énonciation par Ann de l'introduction du discours rapporté $(D o(h) n$ said) ? Ne trouve-t-elle pas peutêtre aussi son origine dans des récits antérieurs ? Ann n'a-t-elle pas déjà raconté, en présence de son mari, à d'autres amis, cette petite anecdote ? L'interaction horizontale de Dan avec la parole de sa femme hic et nunc n'est-elle pas sous-tendue par l'interaction verticale avec du raconté antérieur ? Je ne saurais bien sûr répondre à ces questions. Ce qui retient mon attention, c'est que Goodwin ne (se) les pose pas, fût-ce pour écarter ces possibilités... Le souci pour la parole qui se co-construit ne s'accompagne-t-il pas d'un oubli de la parole déjà dite ? Cette façon de faire me semble récurrente en AC. Prenons un second exemple, empruntée à la très intéressante communication «Organisation séquentielle et configurations syntaxiques de la parole-en-interaction» de notre congrès (occurrence (10)). L'auteur analyse «la configuration en-ligne des trajectoires syntaxiques », et propose l'exemple suivant :

(2) Oui bon la littérature c'est- moi je n'ai jamais tellement aimé mais c'est bien sûr c'est bien si on fait ça

Cet énoncé est décrit fort justement comme exemple de «formatage prospectif-rétrospectif de la structuration syntaxique » : le SN la littérature initialement projeté, dans le tour à détachement gauche, comme apposition au démonstratif sujet $c$ ', se voit ensuite réinterprété comme objet du verbe aimer. On peut regretter que le corpus présenté ne nous fournisse pas le dit immédiatement antérieur: l'adverbe initial oui ne présuppose-t-il pas la confirmation d'un propos précédent, ce qui rendrait compte dans un premier temps de la thématisation dialogique (reprise-écho) du SN la littérature selon la structure la plus accessible (« la littérature c'est- »), qui se voit rectifiée ensuite par la bifurcation syntaxique ? Ce qui me retient ici également, c'est que la présentation comme la segmentation du corpus proposé ne permettent pas de répondre... L'intérêt pour le dire en cours ne va-t-il pas de pair avec le désintérêt pour le déjà dit (ici immédiat)... comme si celui-ci n'en était pas un paramètre déterminant? 
Plus constructivement, j'aimerais pointer, à partir d'un autre exemple, ce que l'approche dialogique peut apporter à l'AC. Goodwin formule une deuxième critique, tout aussi pertinente, à l'encontre tant de Voloshinov que de Goffman : le fait que ces deux auteurs pensent le discours rapporté seulement en termes de complexité syntaxique (enchâssement du discours cité dans le discours citant) écarte de l'analyse des phénomènes qui ont à voir avec le discours rapporté mais dans lesquels ce type de syntaxe ne se manifeste pas. Ce que Goodwin illustre par l'étude d'un fragment d'interaction entre trois personnes : Chil, qui à la suite d'un accident ne peut plus dire que yes, no et and, son fils Chuck et sa belle-fille Candy. Celle-ci parle de la neige qui est tombée avec moins d'abondance cette année que précédemment.

(3)

10 Candy : but last year.whoo !

\begin{tabular}{ll}
11 Chuck & \multicolumn{1}{c}{$\mathrm{mm}$} \\
12 & in the last year- \\
13 Chil: & yeah- no no. no:. \\
14 Candy : & er the year before last \\
15 Chil: & yes
\end{tabular}

En 10 et 12, Candy pose que les chutes de neige ont été fortes l'année dernière (« last year »), datation que Chil en 13 commence par confirmer («yeah»), avant de se reprendre pour l'infirmer ( no no. no:. »). Ce qui entraîne une reformulation de Candy ligne 14 (« the year before last »), nouvelle datation que Chil confirme ligne 15 (« yes »).

Goodwin pointe que Chil, alors qu'il ne peut citer (discours rapporté) les paroles de Candy, est à même de les incorporer dans ses possibilités discursives réduites à l'extrême: la négation de la ligne 13 « indexically incorporates what Candy said in line (12), though Chil does not, and cannot, quote what she said there » (op. cit. : 27). De son côté, Candy, ligne 14, en prononçant " the year before last », alors même qu'elle ne rapporte pas la parole de Chil, parle pour lui : «though not reporting the speech of another, Candy speaks for Chil in (14), and locates him as the Principal for what is being said there" (op. cit.: 28).

Effectivement, l'approche de Voloshinov comme celle de Goffman, en associant discours rapporté et complexité syntaxique, ne sont pas à même de rendre compte de phénomènes comme ceux-ci, où, s'il n'y a pas citation effective par A de la parole de B, il est bien question dans l'énoncé de A de la parole de B. Il me semble que l'approche dialogique, en prolongement des intuitions que l'on trouve dans Bakhtine $(1934,1952)$, permet de proposer un traitement unitaire de ces questions. Le statut particulier accordé au discours rapporté ne tient que pour autant que l'on met l'accent, dans l'analyse, sur la parole rapportée, en effacement, voire en oubli de ce qu'il s'agit avant toute chose de l'interaction entre deux énoncés. Pour l'approche dialogique, il n'y a pas d'un côté le discours rapporté (discours direct, indirect, indirect libre, direct libre, voire discours narrativisé...), et de l'autre des tours syntaxiques (confirmation, négation comme dans l'occurrence (3), mais aussi conditionnel, ironie, détournement, etc.) qui, d'une façon ou d'une autre, ont à voir avec la parole d'un autre énonciateur (Bres et Verine 2003). Dans les deux cas, on a affaire à un énoncé dialogique défini comme structuré autour d'un dialogue interne, à savoir comme le résultat de l'interaction entre deux énonciations (au moins) : celle du locuteurénonciateur qui a la parole, celle d'un autre énonciateur. Les formes de cette interaction interne sont extrêmement variées. C'est donc le même phénomène, à savoir l'interaction dialogique, qui est à la base tant du discours direct - forme la plus explicite de la dualité énonciative - que du type de confirmation, ou de négation que l'on trouve en (3), types dans lesquels ladite dualité est fortement implicitée. La négation prédicative, en tant que marqueur dialogique, présuppose l'énoncé positif correspondant qu'elle 
peut « rapporter » de différentes manières. Soit en reprenant l'occurrence (3) et en l'adaptant librement au français :

(4)

L1 : (il a neigé beaucoup plus) l'année dernière

L2 : a) il n'est pas vrai qu'il a neigé beaucoup plus « l'année dernière » ${ }^{9}$

b) il n'est pas vrai qu'il a neigé beaucoup plus l'année dernière

c) non, ce n'est pas « l'année dernière » qu'il a neigé beaucoup plus

d) non, ce n'est pas l'année dernière qu'il a neigé beaucoup plus

e) ce n'est pas « l'année dernière » qu'il a neigé beaucoup plus

f) ce n'est pas l'année dernière qu'il a neigé beaucoup plus

g) non

h) $\ldots$

Parmi ces différentes possibilités syntaxiques, l'énoncé g), à savoir « non »- seul possible pour Chil du fait de son handicap - est celui qui incorpore le plus fortement l'énoncé positif correspondant avec lequel l'interaction a lieu. Ajoutons que, dans ce cas, l'énoncé positif doit effectivement avoir été actualisé par un autre locuteur (L1), ce qui implique que l'énoncé négatif non, autonome, ne peut apparaître qu'en discours dialogal ${ }^{10}$.

Considérer le discours rapporté comme un fait dialogique comme les autres, permet, en ne se focalisant plus sur lui, de traiter de nombreux phénomènes qui comme lui procèdent de l'interaction verticale avec du discours (éloigné ou immédiatement) antérieur, et interviennent comme paramètres dans la production horizontale du discours en interaction.

Je voudrais pour finir ajouter que la notion bakhtinienne de bivocalité (ou de plurivocalité) permet de rendre compte de faits énonciatifs extrêmement complexes, comme p. ex. ce qui intervient ligne 14 de l'occurrence (3), que je rappelle pour plus de commodité :

(3)

10 Candy : but last year.whoo !

11Chuck

$\mathrm{mm}$

In the last year-

13 Chil:

yeah- no no. no:.

14 Candy :

er the year before last

15 Chil:

yes

Goodwin, nous l'avons vu, propose l'analyse suivante: «though not reporting the speech of another, Candy speaks for Chil in (14), and locates him as the Principal for what is being said there" (op. cit. : 28). On dirait plutôt, en termes dialogiques, que cet énoncé est bivocal, que s'y font entendre deux "voix", ici convergentes: celle de Candy elle-même en correction de son énoncé de la ligne 12, du fait de la négation que lui a opposée Chil en 13 ; celle de Chil, par une sorte de ventriloquisme : Candy verbalise les mots que Chil ne peut actualiser.

Il semble donc que l'analyse conversationnelle a tout intérêt à ne pas limiter sa lecture des écrits du cercle de Bakhtine à Voloshinov 1929 : l'approche dialogique des faits linguistiques, qui permet de prendre en 
compte dans sa complexité la dimension verticale du déjà dit dans la production du dire, n'y apparaît pas encore.

\section{En guise de conclusion : l'épaisseur du discours}

Partant du fait que les communications retenues pour ce congrès relevaient principalement du discours dialogal, qu'elles traitaient dans les cadres de l'AC, nous avons questionné cette approche, en prenant appui sur la notion de dialogisme: les pertinentes descriptions que propose l'AC de la co-construction horizontale du discours ne négligent-elles pas une autre dimension - verticale - du discours ? Ce qui se passe dans l'ici et maintenant du mot à mot dans le face-à-face de l'interaction conversationnelle ne se double-t-il pas constamment de l'interaction avec de l'ailleurs antérieur discursif?

Linéarité du discours? Non, bien plutôt épaisseur; et épaisseur double : épaisseur dialogale, telle que la décrit l'AC ; épaisseur dialogique, telle que l'approche bakhtinienne invite à l'analyser.

\section{Références bibliographiques}

Authier-Revuz, J. (1982). Hétérogénéité montrée et hétérogénéité constitutive. Eléments pour une approche de l'autre dans le discours. DRLAV 26, 91-151.

Bakhtine, M. (1934/1975/1978). Du discours romanesque. Esthétique et théorie du roman, Paris : Gallimard, Tel, 83233.

Bakhtine, M. (1952/1979/1984). Les genres du discours. Esthétique de la création verbale, Paris : Gallimard, 265308.

Bres, J. (2005). Savoir de quoi on parle : dialogal, dialogique, polyphonique, Bres, J., Haillet, P., Mellet, S., Nølke, H., Rosier, L. (éd.), Dialogisme, polyphonie : approches linguistiques, Actes du colloque de Cerisy, 47-62.

Bres, J. et Rosier, L. (2008). Réfractions : polyphonie et dialogisme, deux ensembles de reconfigurations théoriques dans les sciences du langage francophones. Slavica Occitania, à paraître.

Bres, J. et Verine, B. (2003). Le bruissement des voix dans le discours : dialogisme et discours rapporté. Faits de langue 19, 159-170.

Charaudeau, P. et Maingueneau, D. (2002). Dictionnaire d'analyse du discours. Paris : Seuil.

Détrie, C. , Siblot, P. et Verine, B. (éd.). (2001). Termes et concepts pour l'analyse du discours. Une approche praxématique. Paris : Honoré Champion.

Goodwin, Ch. (2007). Interactive footing. Holt, E. et Clift, R. (eds), Reporting talk: Reported Speech in interaction, Cambridge : CUP, 16-46.

Goffman, E. (1981). Footing. Forms of talk, Philadelphia: University of Pennsylvania Press, 124-159.

Moirand, S. (2006). Les discours de la presse quotidienne. Paris : PUF.

Paveau, M. A. et Rosier, L. (2005). Eléments pour une histoire de l'analyse du discours. Théories en conflit et ciment phraséologique. Communication au colloque L'analyse du discours en France et en Allemagne, Créteil, Céditec Paris 12. En ligne sur http://wwwjohannes-angermuller.de/francais/adfa.html.

Rosier, L. (2003). L'approche du discours rapporté par l'analyse du discours. Un bilan critique et une piste de recherche. Amossy, R. et Maingueneau, D., L'analyse du discours dans les études littéraires, Toulouse : Presses universitaires du Mirail, 97-109

Tannen, D. (1989). Talking voices. Cambridge : University Press.

Voloshinov, V. N. (1929/1977). Le marxisme et la philosophie du langage. Paris : Minuit.

1 Prudence cependant: les communications retenues dans ce colloque ne visent pas à la représentativité, et ne sauraient être prises comme telles. Cf. p. ex. les travaux actuels sur le discours de presse de Moirand (2006). 


\footnotetext{
${ }^{2}$ Sans prendre partie dans la querelle de paternité qui entoure certains textes, on emploiera le patronyme Bakhtine comme métonymie de Cercle de Bakhtine.

${ }^{3}$ Cf. p. ex. les deux dictionnaires récents d'analyse du discours (Détrie C., Siblot P., et Verine B. (éd.) 2001 ; Charaudeau P. et Maingueneau D. (éd.) 2002).

${ }^{4}$ Par cette seconde dimension, le dialogique se rapproche du dialogal, mais sans se confondre avec lui : le texte monologal, qui en tant que tel, n'a rien de dialogal, est tout entier traversé par le dialogisme interlocutif (anticipation sur la réponse de l'allocutaire, le plus souvent le lecteur).

${ }^{5}$ Connaissance fort partielle et certainement incomplète : je suis « preneur » d'indications complémentaires.

${ }^{6}$ Exception notable : Tannen 1989.

${ }^{7}$ Pour une analyse de la prise en compte du discours rapporté par l'AD, cf. Rosier 2003.

${ }^{8}$ Le (h) note le rire.

${ }^{9}$ Etant entendu qu'à l'oral la modalisation autonymique que nous avons signifiée ici par les guillemets, se marque de bien des façons : mimo-gestualité des doigts des deux mains, intonation, etc.

${ }^{10}$ Le graffiti, p. ex., qui relève le plus souvent du discours monologal, ne saurait actualiser ce type d'énoncé. Exemples de graffitis lus en Catalogne : «Catalunya no és Espanya » ('La Catalogne n'est pas l'Espagne'), ou « No som espanyols » ('nous ne sommes pas espagnols'). Je n'ai aucune occurrence monologale du type « Non! »... qui serait le fait d'un gauchisme infantile prolongé et appuyé...
} 